\title{
The role of psychosocial determinants in predicting adherence to treatment in patient with hypertension
}

\author{
MOHAMMAD REZA ASGARI ${ }^{1}$, HAMID BOURAGHI ${ }^{2}$, ALI MOHAMMADPOUR ${ }^{2}$, \\ MINA HAGHIGHAT ${ }^{3}$, RAHELEH GHADIRI ${ }^{4, *}$ \\ ${ }^{1}$ Nursing Care Research Center, Semnan University of Medical Sciences, Semnan, Iran \\ ${ }^{2}$ Department of Health Information Technology, School of Paramedical Sciences, \\ Hamadan University of Medical Sciences, Hamadan, Iran \\ ${ }^{3}$ Unit of Psychology Consultation, Semnan University, Semnan, Iran \\ ${ }^{4}$ Jahadieh Health Center, Semnan University of Medical Sciences, Semnan, Iran \\ *Corresponding author: Raheleh Ghadiri; Clinical Psychologist, Jahadieh Health Center, Semnan University of Medical Sciences, Semnan \\ 35198 99951, Iran; Phone: +98 233344 1022; Fax: +98 233344 8999; E-mail: Ghadiriraheleh27@gmail.com
}

(Received: March 10, 2018; Revised manuscript received: July 18, 2018; Accepted: July 19, 2018)

\begin{abstract}
Introduction: Non-adherence in patients with hypertension directly exacerbates clinical outcomes. The purpose of the present research is to study the recognition of the relationships between the perceived social support and self-efficacy and the satisfaction of health care agents and the interaction of the patient with therapeutic personnel and access to health care and the behaviors of adherence to treatment in the patients who suffer hypertension. Materials and methods: This descriptive cross-sectional correlation study recruited 250 patients from a specialized hypertension clinic in Semnan, who completed the following questionnaires: Multidimensional Scale of Perceived Social Support, self-efficacy, adherence to treatment, access to and satisfaction with health care, and the patient's interaction with treatment personnel. Results: An overall statistical description of the sample consists of $89(35.6 \%)$ men and $161(64.4 \%)$ women $(S D=10.41$, range $=51.98)$. Regression coefficient of previous variables (three steps) shows that selfefficacy share, consent form civil services, and job could demonstrate with $99 \%$ certainty in the changes of treatment conformity in a meaningful way. Conclusions: High self-efficacy, satisfaction with health care, and a favorable job have a high direct effect on adherence to treatment in patients with hypertension and controlling hypertension. Social support and education do not have a significant impact on adherence to treatment.
\end{abstract}

Keywords: self-efficacy, social support, health services accessibility, hypertension, patient's compliance

\section{Introduction}

Hypertension is the largest global epidemic disease that threatens health in advanced countries [1]. Blood pressure is the amount of force exerted upon the arterial wall to allow blood flow throughout the body [2-4]. Hypertension causes physiological and biochemical changes in the blood vessel wall, which disrupt blood flow, vascular reconstruction, and endothelial function [5]. Hypertension has a major role in the incidence of coronary artery diseases, acute myocardial infarction, brain stroke and its related events, congestive heart failure, renal failure, and ultimately death. Controlling and treating hypertension aims to significantly reduce mortality and cardiovascular complications [6-8]. Hypertension is the third cause of worldwide mortality and morbidity, which accounts for $31 \%$ of total deaths. According to the $\mathrm{WHO}, 33 \%$ or one out of every three adults over 20 years old have hypertension. This, of course, increases with age $[9,10]$. At least $45 \%$ of ischemic heart diseases and $51 \%-62 \%$ of deaths due to stroke are because of hypertension [11, 12]. The prevalence of arterial hypertension is $20 \%$ in Europe. In the US, 60 million cases were reported in 2000 , whose mean will reach $73 \%$ in 2020 . In Canada, $60 \%$ of patients with hypertension are controlled and treated $[13,14]$. This increases the need to manage the disease. Among the factors influencing the management of the everincreasing

This is an open-access article distributed under the terms of the Creative Commons Attribution-NonCommercial 4.0 International License, which permits unrestricted use, distribution, and reproduction in any medium for non-commercial purposes, provided the original author and source are credited, a link to the CC License is provided, and changes - if any - are indicated. 
hypertension cases is adherence to treatment. Failure to adhere to therapeutic recommendations is a major concern in the management of chronic diseases. Some clear factors in treatment non-adherence that include executive performance, lack of family support, lack of motivation for patient-physician interaction, high cost of available medications, long waiting time for visiting the physician, place of residence, social peer pressure, psychologicalpersonality traits, and alcohol use play important roles in increasing hypertension and reducing self-management and compliance with treatment, which in turn lead to serious problems [14-18]. One of these psychological traits is self-efficacy. The treatment of vascular risk factors requires a change in the behavior and compliance with long-term medical therapies. Physicians and nurses must persuade patients to adopt appropriate methods for changing risk factors for vascular diseases, which require promoting self-efficacy. Bandura believes that a person's behavior is constantly interacting with the environment and personal perception. Self-efficacy is the most fundamental factor in self-recognition, which refers to the person's beliefs in his ability (confidence). It is necessary to organize and implement practical courses required for achieving the goals of successful health management. According to the Bandura's theory, self-efficacy beliefs determine how people feel and think, and motivate their behaviors [19]. The concept of self-efficacy is used in two different ways in research: (1) referring to one's perception of the ability to perform a particular behavior and (2) referring to the ability to deal with, control, or accept potential problems that one may face in a particular activity. According to Ashe and Cllasen and Bandura's social cognitive theory, the function of human behavior has been explained as having three modes of agency and two-dimensional causality and the results of the dynamic interaction of individual factors, such as cognition, emotions, and biological events. The unique interaction between all three dimensions leads to behavior change [20]. Lorig et al. [21] showed that promoting self-efficacy is associated with treatment, physical consequences, psychosocial effects, and control of symptoms, such as fatigue, activity limitation, and the number of examinations conducted by the physician in chronic patients. Promoting self-efficacy behaviors improves lifestyle, individual's ability to control blood pressure, and patientphysician interaction, which in turn can promote health behaviors, treatment adherence, and health at higher levels of self-efficacy $[20,22]$. Health care providers also affect treatment adherence. The interaction between a patient and a physician or health care provider is effective in preventing the reduction of treatment motivation or negative emotions of the disease, will increase patient's understanding of symptoms, and reduces disease complications [23]. In this case, given the lack of knowledge and the lack of treatment adherence, informed physicians will be able to try to have more effective partnerships to facilitate collaboration and interaction between patients and physicians to improve patients' understanding of living conditions, including daily frustrations, challenges, and consequences of failing to healthily manage the disease, health, and quality of life. The attitude of physicians, past experiences of patients, and the knowledge of hypertension are the main factors contributing to adherence to treatment and medication, and treatability [24]. Good communication between the patient and health care providers will improve the acceptance of and compliance with the treatment. In some studies, the goal of health care providers was patients' adherence to treatment and disease control. If health care providers identify patients' excuses for treatment non-adherence, feelings of frustration and helplessness will be reduced. Among the important factors in the treatment adherence of individuals are physical and health care factors, such as patientphysician interaction, access to health care, and satisfaction with it. One of the physical resources in controlling hypertension is the traveled distance and easy access to health centers. According to previous studies, proximity to the health center, interaction with and access to human resources, health and treatment systems of providing financial and therapeutic insurance, and positive relationship between the rate of interaction with the physician are highly associated with adherence to the use of anti-hypertension medications [25]. In addition to the role of self-efficacy and health care, social support has been considered to be the most important feature in the prevention of undesirable effects and psychological pressures. Social support represents the resources provided by others in the social structure. These resources can be emotional or instrumental (physical, material, and informational) that help meet the social needs of the individual, which often protect individuals from stressful side effects. Social support also has a direct and positive impact on health and creates a positive feeling and self-esteem $[20,26,27]$. The researchers have tried to classify various protections: (1) emotional support involves expressing sympathy, attention, and affection towards a person; (2) esteem support is expressing respect, encouraging or agreeing with the views, or feelings of a person; (3) tangible or instrumental support includes direct assistance to a person; (4) information support is to provide advice, suggestions, directions, and feedback to the patient; and (5) network support is providing a sense of membership in a group with common interests and activities [28]. Social support from family or friends who are concerned about the patient's hypertension is helpful in reminding to take medication [29]. Fongwa et al. [30] have investigated effective factors in treatment adherence in hypertensive patients, and found a positive association with aforementioned healthy behaviors, which control and to some extent treat hypertension. According to Breaux-Shropshire et al. [31], self-efficacy plays an important role in adherence to therapeutic behaviors and control of hypertension. According to the results of Flynn et al., more than $59 \%$ of 
participants with high levels of self-efficacy and elevated self-esteem were able to properly manage their hypertension [30]. On the other hand, Gohar et al. showed that the support of family and friends, along with easy access to health care centers and services, often plays an important role in treatment adherence, facilitates care provider guidelines and hypertension control, and improves efficacy and sustainability of treatment and intervention [32]. Gohar et al. [33] indicated that the level of income, occupation, and favorable social status have positive effects on compliance with health and treatment behaviors and adherence to hypertension-control processes. In general, social support, access to health care, and self-efficacy affect treatment adherence in hypertension control. Hence, it is necessary to investigate the contribution of each of these variables to the treatment adherence in hypertension, and their relationship with different cultural and health conditions of Iran compared to other countries. Regarding the research gap, the aim of this study was to investigate the relationship between self-efficacy, perceived social support, and health care provider, such as satisfaction with health care providers, patient interaction with health care personnel, and access to health care services, with adherence to treatment in patients with hypertension. Does it have any?

\section{Patients and Methods}

\section{Methodology}

This descriptive analytical correlational study recruited 250 patients (89 males and 161 females) with hypertension in Semnan, Iran through simple random sampling. The mean and standard deviation (SD) of subjects' age were $51.98 \pm$ 10.41 years, respectively. The samples were selected from hospitals and the majority of the patients were randomly selected from the offices of hypertension specialists. Upon achieving a certification, people with early onset hypertension (not simultaneously as another hypertension), and secondary hypertension coincide with other people's blood pressure, such as kidney, diabetes, etc. The researcher visited the hospitals and offices of hypertension specialists in Semnan to collect the target information. In the case of illiterate patients, questionnaires were completed by the researcher through interviews.

\section{Instrument for collecting data}

Data were collected by Personal Information Questionnaire, Self-Efficacy Hypertension Questionnaire, Multidimensional Scale of Perceived Social Support (MSPSS), and a set of health care questionnaires including access to health services, satisfaction with health services, communication with the physician, and Morisky's Medication Adherence Questionnaire for Hypertension.

\section{Medication Understanding and Use Self-Efficacy Scale}

Self-efficacy measurement uses two subscales to measure partcipants' understanding of and confidence in taking their prescription medications. Self-efficacy refers to the activity and practice of taking medications. In this study, we measure the effectiveness of self-efficacy measures to measure patients' perceptions about their ability to fight their illness. The tool consists of 18 dichotomous (yes/no) items, which assess two subscales, where four items assess the ability to use appropriate medication subscale, and four items assess understanding of patients on how to use medication. Cronbach's $\alpha$ was used to assess the reliability and internal validity of the project [34]. The scale was translated into Persian in Iran and tested on 30 patients with hypertension. Its reliability and validity were also assessed using Cronbach's $\alpha$, which reported a 0.90 reliability and validity for the Persian version.

\section{Social Support Questionnaire}

This study used MSPSS by Zimet et al. [35]. MSPSS consists of 12 items and 3 subscales of the family (4 items), friends (4 items), and important people (4 items). In order to obtain the mean total score of the test, the item scores were summed up and divided by the number of items (12). In addition, to calculate the score for each subscale, the item scores of the subscales were summed up and divided by the number of items (4). A high score on this scale reflects a high level of perceived social support. The alpha coefficient of the whole test was 0.91 and the alpha coefficients of its subscales were between 0.91 and 0.95 . It had a favorable factor and concurrent validity.

\section{Morisky's Medication Adberence Questionnaire}

The questionnaire consists of eight items with two types of responses, dichotomous (yes/no), and 4-point Likert scale (from "never" to "always"). Items 1-7 were yes/no questions, and item 8 was answered using the Likert scale. Cronbach's $\alpha$ was used to approve reliability and validity of the 8-item Morisky's scale dimensions, like its 4-item scale [36].

\section{Access to Health Care Services Scale}

This questionnaire includes three subscales of patient's satisfaction with health services ( 8 items), communication with the physician ( 9 items), and patients' access to health services ( 8 items). The alpha coefficient of all three subscales was 0.90 . 


\section{Demographic Information Questionnaire}

This was a researcher-made questionnaire to collect demographic information, including patient's age, gender, marital status, educational level, and occupation. Marital status was divided into four categories (single, married, divorced, and widowed), education into six categories (illiterate, high school, diploma, associate's degree, bachelor's degree, and master's degree), occupation into three categories (unemployed, employed, and retired), and gender into two categories (male and female).

\section{Statistical analysis}

The quantitative variables were presented as mean \pm SD and compared using multiple linear regression, whereas categorical variables were statistically analyzed by Stepwise Method; SPSS software (version 21) was used for analysis, and $p$ values less than 0.01 were considered significant.

\section{Results}

An overall statistical description of the sample consists of $89(35.6 \%)$ men and $161(64.4 \%)$ women $(\mathrm{SD}=0.41$, range $=51.98)$. The majority of subjects are married (89.2). The highest prevalence is in the level of primary education $(27.6 \%)$ and the lowest frequency is related to the master's degree (2.4). The most frequent job related to housekeeper is $(55.6 \%)$, occupying $50(20.4 \%)$, and retired $60(24.0 \%)$.

In order to test the hypotheses, a correlation table between the variables was presented first, and then multiple regressions were performed. Among these variables, self-efficacy was more important than the other two. It can explain the changes related to treatment adherence of the study sample with a $90 \%$ confidence along with other variables. Ultimately, high self-efficacy will lead to treatment adherence and vice versa. These two variables have a direct relationship. Satisfaction with health services was the next significant variable and lower satisfaction with health services reduces treatment adherence. Occupation was the third significant variable, such that a better occupational status was associated with better treatment adherence. The contrary is also true, lower occupational status will lower the adherence.

The regression coefficient of the predictive variables (three steps) showed that three variables of self-efficacy, satisfaction with health services, and occupation can significantly explain changes in the treatment adherence variable with $99 \%$ confidence. This explanation was obtained through the input of all three variables in the third step of the analysis. As demonstrated in Tables I and $I I$, in the first model, the variable that has the highest relationship with the criterion variable was self-efficacy that was entered into the equation. The correlation coefficient of this variable with treatment adherence was 0.337. This variable was able to predict a significant difference between the variables of treatment adherence, which is significant and positively affects the treatment of the patient. In the second model, after the self-efficacy variable, satisfaction with health services was entered into the equation, and the correlation coefficient of these two variables with treatment adherence was 0.429 , which was significant. The coefficient of determination in the second model shows that these two variables predict 0.184 of treatment adherence changes, and the entry of the satisfaction with health services variable was able to increase the predicting power by 0.445 . In the third model, after the variables of self-efficacy and satisfaction with treatment services, the occupation variable was also included in the equation. The multivariable correlation coefficient in this model was 0.457 , which was significant. The coefficient of determination in the third model showed that these three variables predicted 0.209 of treatment adherence variable changes, and the entry of the duration of illness variable has been able to increase the predicting power by 0.024 . In the fourth model, social support variable entered the equation after the self-efficacy variables and satisfaction with the treatment services and occupation. The multivariable correlation coefficient in this model was 0.474 , which was significant. The coefficient of determination in the fourth model showed that these four variables predicted 0.209 of treatment adherence variable changes, and the entry of the social support variable has been able to increase the predicting power by 0.016 .

\section{Discussion}

This study aimed to investigate the psychosocial factors associated with treatment adherence in patients with hypertension in Semnan, Iran. The results show that the mean scores of the scale and subscales of treatment adherence behavior, such as low-salt diet, physical activity, proper weight and weight control, and smoking cessation in patients with hypertension were moderate and had a positive relationship. In Brazil, Grezzana et al. [37] reported almost similar results in a study with similar scales. Fongwa et al., Hashmi et al., and Natarajan et al. reported high adherence to treatment and medication, adherence to a low-salt diet, exercise, and non-smoking behaviors, resulting in a positive correlation with hypertension control $[30,38-40]$. In addition, according to this study, females are more adherent to treatment than males. Female patients in this study were more concerned with treatment and medication use. According to Kayima et al. and Warren-Findlow et al., females are more 
岁

离苛

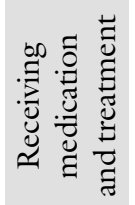

总专莺

호

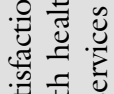

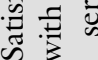

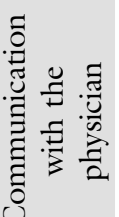

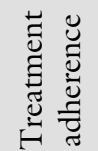

焉.

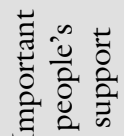

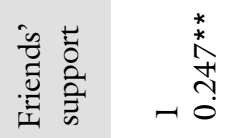

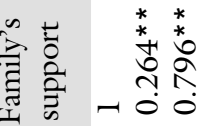

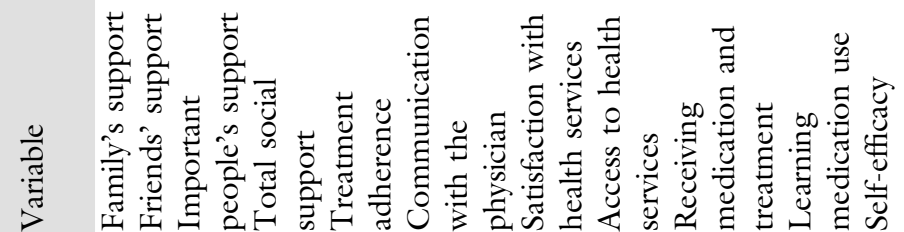

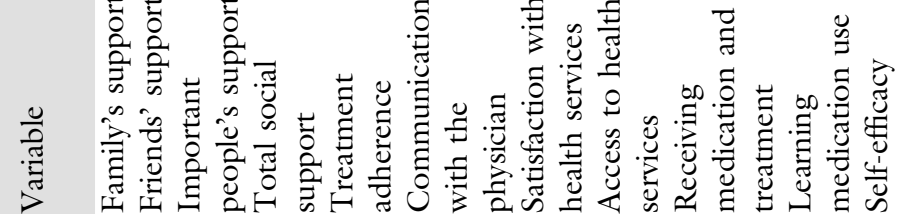

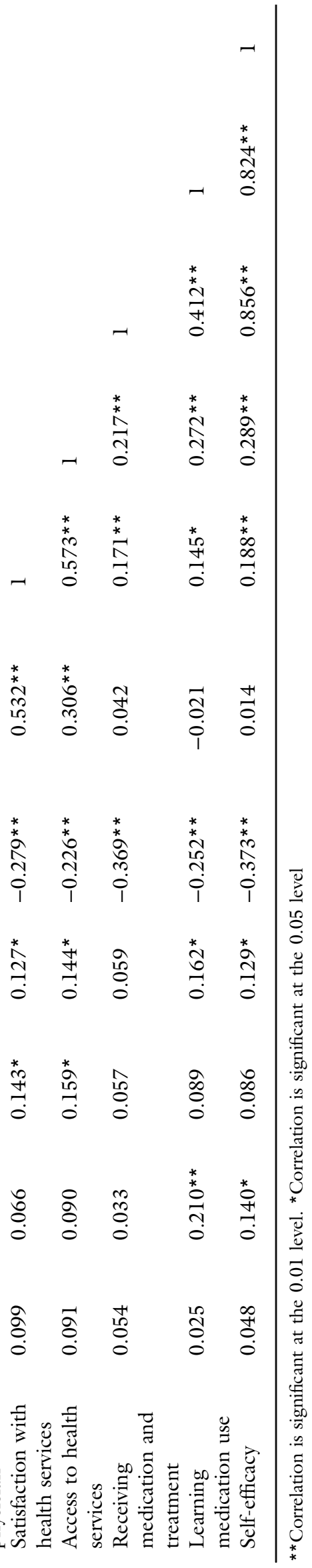


Table II Regression coefficient of predictor variables (three steps) between the three variables of self-efficacy, satisfaction with health services, and occupation on treatment adherence

\begin{tabular}{lrrrc} 
Variable & $B$ & SE $B$ & $\beta$ & $R$ \\
$\begin{array}{l}\text { Total social } \\
\text { support }\end{array}$ & 0.523 & -0.408 & 0.075 & -0.147 \\
$\begin{array}{l}\text { Self-efficacy } \\
\begin{array}{l}\text { Total health } \\
\text { services }\end{array}\end{array}$ & -0.793 & 0.155 & -0.305 & $0.139^{*}$ \\
$\begin{array}{l}\text { Occupation } \\
\begin{array}{l}\text { Satisfaction with } \\
\text { health services }\end{array}\end{array}$ & -0.136 & 0.038 & -0.155 & 0.209 \\
$\begin{array}{l}\text { Medication use } \\
\begin{array}{l}\text { Learning } \\
\text { medication use }\end{array}\end{array}$ & -1.334 & 0.269 & -0.319 & 0.141 \\
$\begin{array}{l}\text { Communication } \\
\text { with the physician }\end{array}$ & 0.081 & 0.040 & 0.145 & 0.089 \\
$\begin{array}{l}\text { Access to health } \\
\text { services }\end{array}$ & -0.042 & 0.031 & -0.099 & 0.089 \\
\hline $\begin{array}{l}\text { SE: standard error. } \\
\text { * Correlation is significant at the } 0.05 \text { level }\end{array}$ & & 0.160 & $0.218^{*}$ \\
\end{tabular}

adherent to medication and treatment than men [41, 42]. According to this study, level of education has no effect on patient's compliance with hypertension treatment. In this study, younger patients were less adherent to treatment than older patients, which is consistent with Hashmi et al. The results of the research in terms of income level and economic status showed that a desirable job affects adherence to treatment behaviors and increases treatment adherence and medication use. Regarding the huge costs of hypertension inhibitor medications, suitable income levels and desirable jobs can play a significant role in acquiring drugs and even compliance with the subscales of treatment adherence behavior and have a positive relationship with treatment. However, Hashmi et al. [38] found no significant difference between income levels. Steptoe et al. [43] indicated that the level of income and a favorable job have positive effects on compliance with health and treatment behaviors and adherence to hypertension-control processes. In this study, the mean of self-efficacy scores was negative. According to the scoring method of the treatment adherence questionnaire, higher treatment adherence scores indicate fewer adherences on the part of the patient. As a result, this negative correlation between self-efficacy and treatment adherence indicates that higher self-efficacy is associated with higher treatment adherence. As a result, self-efficacy scores in this study were high. It can be concluded that self-efficacy enhancement had a positive relationship with overall scores and subgroups of self-efficacy and treatment adherence in hypertensive patients. This was consistent with the results of Warren-Findlow et al., Breaux-Shropshire et al., Savadkooh et al., and Johnel et al. [9, 31, 44-47]. In addition, their study on self-efficacy and treatment adherence obtained similar and positive results. Cameron et al. [34] obtained similar results using similar tools. The amount of support and encouragement that individuals receive about behaviors, such as smoking and exercising, will play an effective role in adherence to treatment. Friends and family encourage or weaken their health behavior by encouraging or punishing, and being a role model or valuing health. Two other factors that affect individual health behaviors are personality and person's mental status. One of the features related to health behaviors is dutifulness. People with a lot of social support have a sense of belonging and self-esteem. As a result, people with high social support may feel that as others value and need them, they should be more concerned with their health. In addition, social support had a negative relationship with the whole scale and subscales scores of social support (friends, relatives, and families). It can be concluded that more than half of the subjects had moderate to poor social support in this study due to the method of scoring of the social support by hypertensive patient's questionnaire. In this study, no positive results were found. The majority of patients have chosen score 4 in scoring $1-4$, which led to negative outcome in the relevant factor analysis. Social support provided by the patients' friends and relatives plays an important role in control and adherence to the treatment of patients, such that their relatives' and families' more attention, concern, and sympathy with the patients increases their selfconfidence, ability, attention to controlling their illness, and adherence to treatment. In contrast to the findings from the studies of Cornwell et al., Deinzer et al.'s, Piwonski et al., Criswell et al., Rodriguez et al., Adeniyi et al., and Redondo-Sendino et al., this study found a direct relationship between social support and adherence to treatment for hypertensive patients [48-54]. That is, increasing or decreasing social support affects treatment adherence, and that adults and older adults need more social support. Among the three factors of health services, including access to medical services, satisfaction with health care providers, and communication with the physician, satisfaction with health services, and access to health services had a significant effect on treatment adherence. The results of this study showed that when patients are more satisfied with and have access to health care services and health insurance to reduce the costs and equipment needed for hypertension, they comply more with treatment adherence behaviors. The results of Baljani et al., Maimarise et al., Mirzaei Alavijeh et al., Johnell et al., Willard-Grace et al., and Khan et al. were consistent with this study and showed a positive relationship between treatment adherence behaviors and selfefficacy of people with hypertension [22, 25, 45, 47, $55,56]$. In addition, Kayaniyil et al. and Gorman et al. showed that high self-efficacy increases adherence to 
treatment and medication in the study subjects and that satisfaction with health services, and the performance of health care providers (physicians) is effective in adherence to treatment and improving hypertension $[57,58]$. This study, which was conducted on hypertensive patients, had some positive features, including the assessment of the role of social support in improving patients with hypertension, which was not investigated in Iran before. Another important feature of this study was the role of health care providers in treatment adherence of patients with hypertension, and no similar study was found in the literature. This study examined the factors of health services and its subscales (satisfaction with health services, communication with the physician, and access to health services).

\section{Conclusions}

High self-efficacy, satisfaction with the health care, and a desired job have had a high direct effect on following the higher treatment of the patients with hypertension and they also have a high relationship with following the treatment and controlling hypertension. In addition, the measures of social support and education level do not have high impact in following the treatment.

Usually, every study has limitations according to its conditions and facilities, which can reduce the scope of its generalizability to the entire community. The lack of a single and specific clinic for patients with hypertension in Semnan was a limitation in this study. Another limitation was the selection of subjects among Semnan patients who may be different in terms of facilities, situations, and problems with those living in smaller towns or villages. It is recommended that further studies examine treatment adherence in villages and cities separately, and the effects of other clinical components on the risk of hypertension with a larger population.

\section{$* * *$}

Funding sources: None.

Authors' contribution: All authors had full access to all data in the study and take responsibility for the integrity of the data and the accuracy of the data analysis.

Conflict of interest: None.

\section{References}

1. Salgado DR, Silva E, Vincent JL: Control of hypertension in the critically ill: A pathophysiological approach. Ann Intensive Care 3, 17 (2013)

2. Hutton B, Tetzlaff J, Yazdi F, Thielman J, Kanji S, Fergusson D, Bjerre L, Mills E, Thorlund K, Tricco A, Straus S, Moher D, Leenen $\mathrm{FH}$ : Comparative effectiveness of monotherapies and combination therapies for patients with hypertension: Protocol for a systematic review with network meta-analyses. Syst Rev 2, 1 (2013)
3. Gomes M, Cobas R, Matheus A, Tannus L, Negrato C, Rodacki M, Braga N, Cordeiro MM, Luescher JL, Berardo RS, Nery M, Arruda-Marques MC, Calliari LE, Noronha RM, Manna TD, Zajdenverg L, Salvodelli R, Penha FG, Foss MC, Foss-Freitas MC, Pires AC, Robles FC, Guedes M, Dib SA, Dualib P, Silva SC, Sepulvida J, Almeida HG, Sampaio E, Rea R, Faria AC, Tschiedel B, Lavigne S, Cardozo GA, Azevedo MJ, Canani LH, Zucatti AT, Coral MH, Pereira DA, Araujo LA, Tolentino M, Pedrosa HC, Prado FA, Rassi N, Araujo LB, Fonseca RM, Guedes AD, Matos OS, Faria M, Azulay R, Forti AC, Façanha C, Montenegro AP, Montenegro R Jr, Melo NH, Rezende KF, Ramos A, Felicio JS, Santos FM, Jezini DL, Cordeiro MM: Regional differences in clinical care among patients with type 1 diabetes in Brazil: Brazilian Type 1 Diabetes Study Group. Diabetol Metab Syndr 4, 1-12 (2012)

4. Babbs C: Oscillometric measurement of systolic and diastolic blood pressures validated in a physiologic mathematical model. Biomed Eng Online 11, 56 (2012)

5. Toque H, Nunes K, Rojas M, Bhatta A, Yao L, Xu Z, Yao L, Xu Z, Romero MJ, Webb RC, Caldwell RB, Caldwell RW: Arginase 1 mediates increased blood pressure and contributes to vascular endothelial dysfunction in deoxycorticosterone acetate-salt hypertension. Front Immunol 4, 44 (2015)

6. Mentz G, Schulz A, Mukherjee B, Ragunathan T, Perkins D, Israel B: Hypertension: Development of a prediction model to adjust selfreported hypertension prevalence at the community level. BMC Health Serv Res 12, 312 (2012)

7. Eisenberg J (2007): AHRQ Comparative Effectiveness Reviews Effectiveness of Self-Measured Blood Pressure Monitoring in Adults with Hypertension. Comparative Effectiveness Review Summary Guides for Clinicians. Agency for Healthcare Research and Quality (US), Rockville, MD

8. Wang J, Xiong X, Yang G, Zhang Y, Liu Y, Zhang Y, Zhang Z, Li J, Yang X: Chinese herbal medicine qi ju di huang wan for the treatment of essential hypertension: A systematic review of randomized controlled trials. Evid Based Complement Alternat Med 2013, 262685 (2013)

9. Warren-Findlow J, Seymour RB, Brunner Huber LR: The association between self-efficacy and hypertension self-care activities among African American adults. J Community Health 37, 15-24 (2012)

10. Mohammadi M, Dadkhah B, Sazavar H, Mozaffari N: The effect of follow up on blood pressure control in hypertensive patients. J Ardabil Univ Med Sci 6, 156-162 (2006)

11. Adebayo RA, Balogun MO, Adedoyin RA, Obashoro-John OA, Bisiriyu LA, Abiodun OO: Prevalence of hypertension in three rural communities of Ife North Local Government Area of Osun State, South West Nigeria. Int J Gen Med 6, 863-868 (2013)

12. Hagins M, States R, Selfe T, Innes K: Effectiveness of yoga for hypertension: Systematic review and meta-analysis. Evid Based Complement Altern Med 2013, 649836 (2013)

13. Nolan RP, Liu S, Feldman R, Dawes M, Barr S, Lynn H, GwardySridhar F, Thomas SG, Goodman J, Oh P, Kaczorowski J, Chessex C, Hachinski V, Shoemaker K: Reducing risk with e-based support for adherence to lifestyle change in hypertension (REACH): Protocol for a multicentred randomised controlled trial. BMJ Open 3, e003547 (2013)

14. Parati G, Omboni S, Compare A, Grossi E, Callus E, Venco A, Destro M, Villa G, Palatini P, Rosei EA, Scalvini S, Taddei S, Manfellotto D, Favale S, De Matteis C, Guglielmi M, TELEBPMET Study Group, Lonati L, Della Rosa F, Tosazzi E, Grandi AM, Maresca AM, Mongiardi C, Mare M, Ricci AR, Cagnoni F, Georgatos J, Besostri V, Ferrari V, Omodeo O, Dorigatti F, Bonso E, Guarnieri C, Muiesan L, Paini A, Stassaldi D, Cinelli A, Bernocchi P, Rocchi S, Magagna A, Ghiadoni L, Del Frate I, Boresi F, Guidi A, Re MA, Pellicciotti L, Florio A, Morani G, Di Lillo S, Ambrosio A, Casciello A, Quaglia M, Forleo C, Ardito MA, Gerunda S, Panunzio M: Blood pressure control and treatment 
adherence in hypertensive patients with metabolic syndrome: Protocol of a randomized controlled study based on home blood pressure telemonitoring vs. conventional management and assessment of psychological determinants of adherence (TELEBPMET Study). Trials 14, 22 (2013)

15. Pyatak EA, Florindez D, Weigensberg MJ: Adherence decision making in the everyday lives of emerging adults with type 1 diabetes. Patient Prefer Adherence 7, 709-718 (2013)

16. Martin MY, Person SD, Kratt P, Prayor-Patterson H, Kim Y, Salas M, Pisu M: Relationship of health behavior theories with selfefficacy among insufficiently active hypertensive African-American women. Patient Educ Couns 72, 137-145 (2008)

17. Rolnick SJ, Pawloski PA, Hedblom BD, Asche SE, Bruzek RJ: Patient characteristics associated with medication adherence. Clin Med Res 11, 54-65 (2013)

18. Valinejadi A, Sadoughi F, Salehi M: Diabetes knowledge translation status in developing countries: A mixed method study among diabetes researchers in case of Iran. Int J Prev Med 7, 33 (2016)

19. Tsang SK, Hui EK, Law BC: Self-efficacy as a positive youth development construct: A conceptual review. ScientificWorldJournal 2012, 452327 (2012)

20. Khalili F, Eslami AA, Farjzadegn Z, Hasanzadeh A: The association between social-psychological factors and treatment adherence behaviors among maintenance hemodialysis patients in Isfahan, Iran: A conceptual framework based on social cognitive theory. J Res Syst Health 7, 278-290 (2010)

21. Lorig K, Chastain RL, Ung E, Shoor S, Holman HR: Development and evaluation of a scale to measure perceived self-efficacy in people with arthritis. Arthritis Rheum 32, 37-44 (1989)

22. Baljani E, Rahimi JH, Amanpour E, Salimi S, Parkhashjoo M: Effects of a nursing intervention on improving self-efficacy and reducing cardiovascular risk factors in patients with cardiovascular diseases. J Fac Nurs Midwifery 17, 45-54 (2011)

23. Sol BG, van der Bijl JJ, Banga JD, Visseren FL: Vascular risk management through nurse-led self-management programs. J Vasc Nurs 23, 20-24 (2005)

24. Saleem F, Hassali MA, Shafie AA, Ul Haq N, Farooqui M, Aljadhay $\mathrm{H}$, Ahmad FU: Pharmacist intervention in improving hypertensionrelated knowledge, treatment medication adherence and healthrelated quality of life: A non-clinical randomized controlled trial. Health Expect 18, 1270-1281 (2015)

25. Maimaris W, Paty J, Perel P, Legido-Quigley H, Balabanova D, Nieuwlaat R, McKee M: The influence of health systems on hypertension awareness, treatment, and control: A systematic literature review. PLoS Med 10, el001490 (2013)

26. Lu Y: Household migration, social support, and psychosocial health: The perspective from migrant-sending areas. Soc Sci Med $74,135-142(2012)$

27. Lino VT, Portela MC, Camacho LA, Atie S, Lima MJ: Assessment of social support and its association to depression, self-perceived health and chronic diseases in elderly individuals residing in an area of poverty and social vulnerability in Rio de Janeiro city, Brazil. PLoS One 8, e71712 (2013)

28. Sarafino EP, Smith TW (2014): Health Psychology: Biopsychosocial Interactions. Wiley, Hoboken, NJ

29. Osamor PE, Owumi BE: Factors associated with treatment compliance in hypertension in southwest Nigeria. J Health Popul Nutr 29, 619-628 (2011)

30. Fongwa MN, Evangelista LS, Hays RD, Martins DS, Elashoff D, Cowan MJ, Morisky DE: Adherence treatment factors in hypertensive African American women. Vasc Health Risk Manag 4, 157-166 (2008)

31. Breaux-Shropshire TL, Brown KC, Pryor ER, Maples EH: Relationship of blood pressure self-monitoring, medication adherence, self-efficacy, stage of change, and blood pressure control among municipal workers with hypertension. Workplace Health Saf 60 , 303-311 (2012)
32. Flynn SJ, Ameling JM, Hill-Briggs F, Wolff JL, Bone LR, Levine DM, Roter DL, Lewis-Boyer L, Fisher AR, Purnell L, Ephraim PL, Barbers J, Fitzpatrick SL, Albert MC, Cooper LA, Fagan PJ, Martin D, Ramamurthi HC, Boulware LE: Facilitators and barriers to hypertension self-management in urban African Americans: Perspectives of patients and family members. Patient Prefer Adherence 7, 741-749 (2013)

33. Gohar F, Greenfield SM, Beevers DG, Lip GY, Jolly K: Self-care and adherence to medication: A survey in the hypertension outpatient clinic. BMC Complement Altern Med 8, 4 (2008)

34. Cameron KA, Ross EL, Clayman ML, Bergeron AR, Federman AD, Bailey SC, Davis TC, Wolf MS: Measuring patients' selfefficacy in understanding and using prescription medication. Patient Educ Couns 80, 372-376 (2010)

35. Zimet GD, Dahlem NW, Zimet SG, Farley GK: The multidimensional scale of perceived social support. J Pers Assess 52, 30-41 (1988)

36. Morisky DE, Ang A, Krousel-Wood M, Ward HJ: Predictive validity of a medication adherence measure in an outpatient setting. J Clin Hypertens (Greenwich) 10, 348-354 (2008)

37. Grezzana GB, Stein AT, Pellanda LC: Blood pressure treatment adherence and control through 24-hour ambulatory monitoring. Arq Bras Cardiol 100, 335-361 (2013)

38. Hashmi SK, Afridi MB, Abbas K, Sajwani RA, Saleheen D, Frossard PM, Ishaq M, Ambreen A, Ahmad U: Factors associated with adherence to anti-hypertensive treatment in Pakistan. PLoS One 2, e280 (2007)

39. Mukora-Mutseyekwa FN, Chadambuka EM: Drug adherence behavior among hypertensive out-patients at a tertiary health institution in Manicaland province, Zimbabwe, 2011. Patient Prefer Adherence 7, 65-70 (2013)

40. Natarajan N, Putnam W, Van Aarsen K, Beverley Lawson K, Burge F: Adherence to antihypertensive medications among family practice patients with diabetes mellitus and hypertension. Can Fam Physician 59, e93-e100 (2013)

41. Kayima J, Wanyenze RK, Katamba A, Leontsini E, Nuwaha F: Hypertension awareness, treatment and control in Africa: A systematic review. BMC Cardiovasc Disord 13, 54 (2013)

42. Warren-Findlow J, Basalik DW, Dulin M, Tapp H, Kuhn L: Preliminary validation of the Hypertension Self-Care Activity Level Effects (H-SCALE) and clinical blood pressure among patients with hypertension. J Clin Hypertens (Greenwich) $15,637-643$ (2013)

43. Steptoe A, Willemsen G: The influence of low job control on ambulatory blood pressure and perceived stress over the working day in men and women from the Whitehall II cohort. J Hypertens 22, 915-920 (2004)

44. Savadkooh OK, Zakerimoghadam M, Gheyasvandian S, Kazemnejad A: Effect of self-management program on self-efficacy in hypertensive patients. J Mazand Univ Med Sci 22, 19-28 (2012)

45. Johnell K, Rastam L, Lithman T, Sundquist J, Merlo J: Low adherence with antihypertensives in actual practice: The association with social participation - A multilevel analysis. BMC Public Health $5,17(2005)$

46. Mirzaei Alavijeh M, Nasirzadeh M, Jalilian F, Mostafavei F, Hafezi M: Self-efficacy of health promotion behaviors in hypertensive patients. Daneshvar (medicine) Shahed Univ 19, $1-8(2012)$

47. Hacihasanoglu R, Gozum S, Capik C: Validity of the Turkish version of the Medication Adherence Self-Efficacy Scale-Short Form in hypertensive patients. Anadolu Kardiyol Derg 12, 241-248 (2012)

48. Cornwell EY, Waite LJ: Social network resources and management of hypertension. J Health Soc Behav 53, 215-231 (2012)

49. Piwonski J, Piwonska A, Sygnowska E: Is level of social support associated with health behaviours modifying cardiovascular risk? Results of the WOBASZ study. Kardiol Pol 70, 803-809 (2012) 
50. Criswell TJ, Weber CA, Xu Y, Carter BL: Effect of self-efficacy and social support on adherence to antihypertensive drugs. Pharmacotherapy 30, 432-441 (2010)

51. Rodriguez CJ, Burg MM, Meng J, Pickering TG, Jin Z, Sacco RL, Boden-Albala B, Homma S, Di Tullio MR: Effect of social support on nocturnal blood pressure dipping. Psychosom Med 70, 7-12 (2008)

52. Adeniyi A, Idowu O, Ogwumike O, Adeniyi C: Comparative influence of self-efficacy, social support and perceived barriers on low physical activity development in patients with type 2 diabetes, hypertension or stroke. Ethiop J Health Sci 22, 113-119 (2012)

53. Redondo-Sendino A, Guallar-Castillon P, Banegas JR, RodriguezArtalejo F: Relationship between social network and hypertension in older people in Spain. Rev Esp Cardiol 58, 1294-1301 (2005)

54. Deinzer A, Babel H, Veelken R, Kohnen R, Schmieder RE: Shared decision-making with hypertensive patients. Results of an implementation in Germany. Dtsch Med Wochenschr 131, 2592-2596 (2006)
55. Willard-Grace R, DeVore D, Chen EH, Hessler D, Bodenheimer T, Thom DH: The effectiveness of medical assistant health coaching for low-income patients with uncontrolled diabetes, hypertension, and hyperlipidemia: Protocol for a randomized controlled trial and baseline characteristics of the study population. BMC Fam Pract 14, 27 (2013)

56. Khan MA, Javed W, Ahmed M, Walley J, Khan HJ: Delivering enhanced cardiovascular (hypertension) disease care through private health facilities in Pakistan. BMC Cardiovasc Disord 13, 76 (2013)

57. Kayaniyil S, Gravely-Witte S, Stewart DE, Higginson L, Suskin N, Alter D, Grace SL: Degree and correlates of patient trust in their cardiologist. J Eval Clin Pract 15, 634-640 (2009)

58. Gorman BK, Sivaganesan A: The role of social support and integration for understanding socioeconomic disparities in self-rated health and hypertension. Soc Sci Med 65, 958-975 (2007) 